\title{
Surface and bulk acoustic waves in two-dimensional phononic crystal consisting of materials with general anisotropy
}

\author{
Tsung-Tsong $\mathrm{Wu}, *$ Zi-Gui Huang, and S. Lin \\ Ultrasonics Lab., Institute of Applied Mechanics, National Taiwan University, Taipei 106, Taiwan \\ (Received 15 July 2003; revised manuscript received 15 October 2003; published 10 March 2004)
}

\begin{abstract}
Successful application of photonic crystals has led recently to a rapidly growing interest in the analogous acoustic effects in periodic elastic structures called phononic crystals. This study is aimed at developing a theory for two-dimensional phononic crystal consisting of materials with general anisotropy. Explicit formulations of the plane harmonic bulk wave and the surface wave dispersion relations in such a general phononic structure are derived based on the plane wave expansion method. Two-dimensional phononic structures with either the square or the hexagonal lattice are considered in the numerical examples. Band gap characteristics of the phononic structures with different anisotropic background materials (isotropic, cubic, hexagonal, and orthorhombic) are calculated and discussed.
\end{abstract}

DOI: 10.1103/PhysRevB.69.094301

PACS number(s): 43.20.+g, 43.35.+d, 43.40.+s, 46.40.Cd

\section{INTRODUCTION}

The existence of complete band gaps of electromagnetic waves in photonic structures extending throughout the Brillouin zone has demonstrated a variety of fundamental and practical interests. ${ }^{1,2}$ This has led to a rapidly growing interest in the analogous acoustic effects in periodic elastic structures called the phononic crystals. Surface wave propagation on layered superlattices with traction free surface parallel to the layers has been explored extensively in the past. ${ }^{3}$ However, investigations on surface wave properties of solids in which the periodic modulation occurs on the traction free surface has not started until recently. ${ }^{4-8}$ Vinces et al. ${ }^{4,5}$ studied experimentally the surface waves generated by a linefocus acoustic lens at the water-loaded surfaces of a number of two-dimensional superlattices that intersect the surface normally. Propagation of Scholte-like acoustic waves at the liquid-loaded surfaces of period structures has also been studied. $^{6}$

The superlattices considered in Refs. 4-6, 8 are made of isotropic materials. As for superlattices consist of anisotropic materials, Tanaka and Tamura ${ }^{7}$ reported detail calculations for surface waves on a square superlattice consisting of cubic materials (AlAs/GaAs) and many salient features of surface waves in two-dimensional superlattices have been described. In addition, Tanaka and Tamura ${ }^{8}$ also reported detail calculations for surface waves on a hexagonal superlattice consisting of isotropic materials ( $\mathrm{Al} /$ polymer).

Analyses of bulk acoustic waves in phononic structures consisted of isotropic materials have been conducted and reported in literatures. ${ }^{9-15}$ Three different schemes were usually adopted in the calculation, i.e., the plane wave expansion method, the multiple scattering method, and the finite difference time domain method. Kushwaha et al. ${ }^{9,10}$ utilized the plane wave expansion method to calculate the first full band structure of the transverse polarization mode for periodic, elastic composite and further, calculated the band structures for the transverse polarization modes of nickel alloy cylinders in aluminum alloy host. In Refs. 11-14, the multiple scattering theory was applied to study the band gaps of three- dimensional periodic acoustic composites and the band structure of a phononic crystal consisting of complex and frequency-dependent Lamé coefficients. Garcia-Pablos et al. ${ }^{15}$ used the finite difference time domain method to interpret the experimental data of two-dimensional systems consisting of cylinders of fluids $(\mathrm{Hg}$, air, and oil) inserted periodically in a finite slab of $\mathrm{Al}$ host.

In this paper, we extend Tanaka and Tamura's work ${ }^{7}$ to study phononic band gaps of surface waves in twodimensional phononic structures consist of general anisotropic materials. The explicit formulations of the plane harmonic bulk wave and the surface wave dispersion relations in such a general phononic structure are derived based on the plane wave expansion method. Two-dimensional phononic structures with either the square or the hexagonal lattice are considered in the numerical examples. Band gap characteristics of the phononic structures with different anisotropic background materials (isotropic, cubic, hexagonal, and orthorhombic) are calculated and discussed.

\section{EQUATIONS OF MOTION OF 2D PHONONIC CRYSTALS}

In an inhomogeneous linear elastic anisotropic medium with no body force, the equation of motion for the displacement vector $\mathbf{u}(\mathbf{r}, t)$ can be written as

$$
\rho(\mathbf{r}) \ddot{u}_{i}(\mathbf{r}, t)=\partial_{j}\left[C_{i j m n}(\mathbf{r}) \partial_{n} u_{m}(\mathbf{r}, t)\right],
$$

where $\mathbf{r}=(\mathbf{x}, z)=(x, y, z)$ is the position vector, $\rho(\mathbf{r})$, $C_{i j m n}(\mathbf{r})$ are the position-dependent mass density and elastic stiffness tensor, respectively. In the following, we consider a phononic crystal composed of a two dimensional periodic array ( $x-y$ plane) of material $A$ embedded in a background material $B$. Both materials $A$ and $B$ are crystals with the lowest symmetry, i.e., belonging to the triclinic symmetry. Due to the spatial periodicity, the material constants, $\rho(\mathbf{x})$, $C_{i j m n}(\mathbf{x})$ can be expanded in the Fourier series with respect to the two-dimensional reciprocal lattice vectors (RLV), G $=\left(G_{1}, G_{2}\right)$, as 


$$
\begin{gathered}
\rho(\mathbf{x})=\sum_{\mathbf{G}} e^{i \mathbf{G} \cdot \mathbf{x}} \rho_{\mathbf{G}}, \\
C_{i j m n}(\mathbf{x})=\sum_{\mathbf{G}} e^{i \mathbf{G} \cdot \mathbf{x}} C_{\mathbf{G}}^{i j m n},
\end{gathered}
$$

where $\rho_{\mathbf{G}}$ and $C_{\mathbf{G}}^{i j m n}$ are the corresponding Fourier coefficients and are defined as

$$
\begin{gathered}
\rho_{\mathbf{G}}=A_{c}^{-1} \int d^{2} x \rho(\mathbf{x}) e^{-i \mathbf{G} \cdot \mathbf{x}}, \\
C_{\mathbf{G}}^{i j m n}=A_{c}^{-1} \int d^{2} x C_{i j m n}(\mathbf{x}) e^{-i \mathbf{G} \cdot \mathbf{x}} .
\end{gathered}
$$

In the above equations, $A_{c}$ is the area of the primitive unit cell of a two-dimensional phononic structure. On utilizing the Bloch theorem and expanding the displacement vector $\mathbf{u}(\mathbf{r}, t)$ in Fourier series, we have

$$
\mathbf{u}(\mathbf{r}, t)=\sum_{\mathbf{G}} e^{i \mathbf{k} \cdot \mathbf{x}-i \omega t}\left(e^{i \mathbf{G} \cdot \mathbf{x}} \mathbf{A}_{\mathbf{G}} e^{i k_{z} z}\right)
$$

where $\mathbf{k}=\left(k_{1}, k_{2}\right)$ is the Bloch wave vector, $\omega$ is the circular frequency, $k_{z}$ is the wave number along the $z$ direction, and $\mathbf{A}_{\mathbf{G}}$ is the amplitude of the displacement vector. We note that as the component of the wave vector $k_{z}$ equals to zero, Eq. (6) degenerates into the displacement vector of a bulk acoustic wave.

Substituting Eqs. (2), (3) and (6) into Eq. (1), and after collecting terms systematically, we obtain

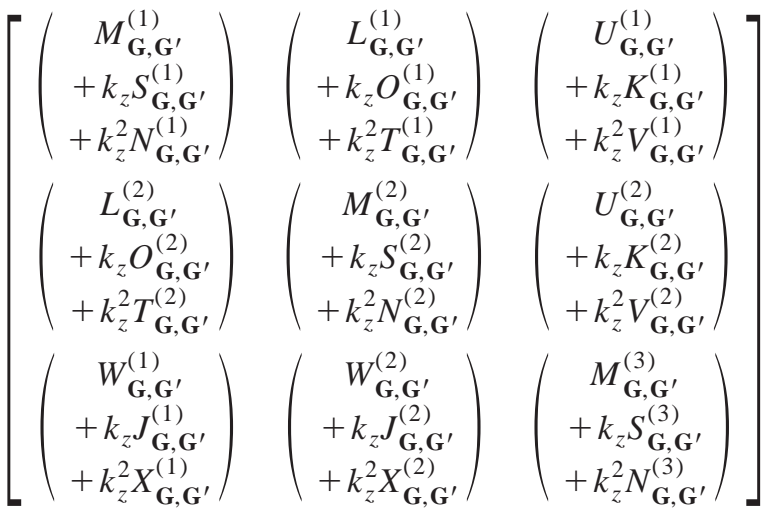

$$
\begin{aligned}
& \cdot\left[\begin{array}{c}
A_{\mathbf{G}^{\prime}}^{1} \\
A_{\mathbf{G}^{\prime}}^{2} \\
A_{\mathbf{G}^{\prime}}^{3}
\end{array}\right]=0,
\end{aligned}
$$

where the $n \times n$ matrices $M_{\mathbf{G}, \mathbf{G}^{\prime}}^{(1)}, M_{\mathbf{G}, \mathbf{G}^{\prime}}^{(2)}, M_{\mathbf{G}, \mathbf{G}^{\prime}}^{(3)}, S_{\mathbf{G}, \mathbf{G}^{\prime}}^{(1)}$, etc. are functions of the Bloch wave vector $\mathbf{k}$, components of the two-dimensional RLV, circular frequency $\omega$, the Fourier coefficients of mass density $\rho_{\mathbf{G}}$ and components of elastic stiffness tensor $C_{\mathbf{G}}^{i j m n}$. $n$ is the total number of RLV used in the Fourier expansion. The expressions of the 27 matrices, $M_{\mathbf{G}, \mathbf{G}^{\prime}}^{(1)}, M_{\mathbf{G}, \mathbf{G}^{\prime}}^{(2)}, M_{\mathbf{G}, \mathbf{G}^{\prime}}^{(3)}, S_{\mathbf{G}, \mathbf{G}^{\prime}}^{(1)}$, etc., in Eq. (7) are listed in the Appendix.
The form of Eq. (7) can be rewritten in the form of a generalized eigenvalue problem with respect to $k_{z}$ as

$$
\left(\mathbf{A} k_{z}^{2}+\mathbf{B} k_{z}+\mathbf{C}\right) \cdot \mathbf{U}=0
$$

where

$$
\begin{gathered}
\mathbf{A}=\left[\begin{array}{ccc}
N_{\mathbf{G}, \mathbf{G}^{\prime}}^{(1)} & T_{\mathbf{G}, \mathbf{G}^{\prime}}^{(1)} & V_{\mathbf{G}, \mathbf{G}^{\prime}}^{(1)} \\
T_{\mathbf{G}, \mathbf{G}^{\prime}}^{(2)} & N_{\mathbf{G}, \mathbf{G}^{\prime}}^{(2)} & V_{\mathbf{G}, \mathbf{G}^{\prime}}^{(2)} \\
X_{\mathbf{G}, \mathbf{G}^{\prime}}^{(1)} & X_{\mathbf{G}, \mathbf{G}^{\prime}}^{(2)} & N_{\mathbf{G}, \mathbf{G}^{\prime}}^{(3)}
\end{array}\right], \\
\mathbf{B}=\left[\begin{array}{lll}
S_{\mathbf{G}, \mathbf{G}^{\prime}}^{(1)} & O_{\mathbf{G}, \mathbf{G}^{\prime}}^{(1)} & K_{\mathbf{G}, \mathbf{G}^{\prime}}^{(1)} \\
O_{\mathbf{G}, \mathbf{G}^{\prime}}^{(2)} & S_{\mathbf{G}, \mathbf{G}^{\prime}}^{(2)} & K_{\mathbf{G}, \mathbf{G}^{\prime}}^{(2)} \\
J_{\mathbf{G}, \mathbf{G}^{\prime}}^{(1)} & J_{\mathbf{G}, \mathbf{G}^{\prime}}^{(2)} & S_{\mathbf{G}, \mathbf{G}^{\prime}}^{(3)}
\end{array}\right], \\
\mathbf{C}=\left[\begin{array}{lll}
M_{\mathbf{G}, \mathbf{G}^{\prime}}^{(1)} & L_{\mathbf{G}, \mathbf{G}^{\prime}}^{(1)} & U_{\mathbf{G}, \mathbf{G}^{\prime}}^{(1)} \\
L_{\mathbf{G}, \mathbf{G}^{\prime}}^{(2)} & M_{\mathbf{G}, \mathbf{G}^{\prime}}^{(2)} & U_{\mathbf{G}, \mathbf{G}^{\prime}}^{(2)} \\
W_{\mathbf{G}, \mathbf{G}^{\prime}}^{(1)} & W_{\mathbf{G}, \mathbf{G}^{\prime}}^{(2)} & M_{\mathbf{G}, \mathbf{G}^{\prime}}^{(3)}
\end{array}\right],
\end{gathered}
$$

and

$$
\mathbf{U}=\left[\begin{array}{c}
A_{\mathbf{G}^{\prime}}^{1} \\
A_{\mathbf{G}^{\prime}}^{2} \\
A_{\mathbf{G}^{\prime}}^{3}
\end{array}\right] .
$$

Equation (8) is more complicated than that of the twodimensional phononic crystal with cubic symmetry given by Tanaka and Tamura ${ }^{7}$ in such a way that the coefficient matrix $\mathbf{B}$ is not vanished. However, it can be solved by introducing $\mathbf{V}=k_{z} \mathbf{U}$ and rewritten in the form as ${ }^{16}$

$$
\left[\begin{array}{cc}
\mathbf{0} & \mathbf{I} \\
-\mathbf{A}^{-1} \mathbf{C} & -\mathbf{A}^{-1} \mathbf{B}
\end{array}\right]\left[\begin{array}{l}
\mathbf{U} \\
\mathbf{V}
\end{array}\right]=k_{z}\left[\begin{array}{l}
\mathbf{U} \\
\mathbf{V}
\end{array}\right] .
$$

\section{BULK AND SURFACE WAVES IN 2D PHONONIC CRYSTALS}

It is worth noting that the case of bulk wave is a special case of Eq. (8). When $k_{z}$ in Eq. (8) is equal to zero, the equation degenerates into the eigenvalue problem of bulk waves as

$$
\mathbf{C} \cdot \mathbf{U}=\mathbf{0} \text {. }
$$

The dispersion relations of bulk waves propagating in twodimensional phononic crystals with both the filling material and the background material belong to the triclinic system, can be obtained by setting the determinant of matrix $\mathbf{C}$ equal to zero.

For material with symmetry higher (and equal to) than orthorhombic symmetry, the components $U_{\mathbf{G}, \mathbf{G}^{\prime}}^{(1)}, U_{\mathbf{G}, \mathbf{G}^{\prime}}^{(2)}$, $W_{\mathbf{G}, \mathbf{G}^{\prime}}^{(1)}, W_{\mathbf{G}, \mathbf{G}^{\prime}}^{(2)}$ in matrix $\mathbf{C}$ are zero and Eq. (14) can be decoupled into two different polarization modes as 


$$
\left[\begin{array}{cc}
M_{\mathbf{G}, \mathbf{G}^{\prime}}^{(1)} & L_{\mathbf{G}, \mathbf{G}^{\prime}}^{(1)} \\
L_{\mathbf{G}, \mathbf{G}^{\prime}}^{(2)} & M_{\mathbf{G}, \mathbf{G}^{\prime}}^{(2)}
\end{array}\right]\left[\begin{array}{c}
A_{\mathbf{G}^{\prime}}^{1} \\
A_{\mathbf{G}^{\prime}}^{2}
\end{array}\right]=0
$$

for mixed polarization modes [i.e., longitudinal (L) and shear horizontal (SH)] and

$$
\left[M_{\mathbf{G}, \mathbf{G}^{\prime}}^{(3)}\right]\left[A_{\mathbf{G}^{\prime}}^{3}\right]=0
$$

for shear vertical (SV) modes with polarization of the displacement along the $z$ direction (i.e., the filler's length direction).

For the case of surface wave, the $6 n$ eigenvalues $k_{z}^{(l)}$ of Eq. (13) are the apparent wave numbers of the plane waves in the $z$ direction. According to the exponential dependence of $z$ in Eq. (6), the real part of $k_{z}^{(l)}$ denotes the plane wave propagation in the $z$ direction, and a positive nonvanishing imaginary part represents attenuation in the $z$ direction. For surface waves propagate in a half space $(z>0)$, only $3 n$ eigenvalues, which attenuate in the positive $z$ direction are chosen, i.e., $\operatorname{Im}\left(k_{z}^{(l)}\right)>0$. Accordingly, the surface wave displacement can be expressed as

$$
\begin{aligned}
\mathbf{u}(\mathbf{r}, t) & =\sum_{\mathbf{G}}{ }^{\prime} e^{i(\mathbf{k}+\mathbf{G}) \cdot \mathbf{x}-i \omega t}\left(\sum_{l=1}^{3 n} \mathbf{A}_{\mathbf{G}} e^{i k_{z}^{(l)} z}\right) \\
& =\sum_{\mathbf{G}}{ }^{\prime} e^{i(\mathbf{k}+\mathbf{G}) \cdot \mathbf{x}-i \omega t}\left(\sum_{l=1}^{3 n} X_{l} \boldsymbol{\varepsilon}_{\mathbf{G}}^{(l)} e^{i k_{z}^{(l)} z}\right),
\end{aligned}
$$

where $\boldsymbol{\varepsilon}_{\mathbf{G}}^{(l)}$ is the associated eigenvector of the eigenvalue $k_{z}^{(l)}$. The prime of the summation denotes that the sum over $\mathbf{G}$ is truncated up to $n . X_{l}$ is the undetermined weighting coefficient which can be determined from the traction free boundary conditions on the surface $z=0$, i.e.,

$$
\left.\left.\mathbf{T}_{i 3}\right|_{z=0} \equiv C_{i 3 m n} \partial_{n} \mathbf{u}_{m}\right|_{z=0}=0 \quad(i=1,2,3) .
$$

Substituting Eq. (17) into Eq. (18), we have

$$
\left[\begin{array}{cccc}
H_{1, \mathbf{G}}^{(1)} & H_{1, \mathbf{G}}^{(2)} & \cdots & H_{1, \mathbf{G}}^{(3 n)} \\
H_{2, \mathbf{G}}^{(1)} & H_{2, \mathbf{G}}^{(2)} & \cdots & H_{2, \mathbf{G}}^{(3 n)} \\
H_{3, \mathbf{G}}^{(1)} & H_{3, \mathbf{G}}^{(2)} & \cdots & H_{3, \mathbf{G}}^{(3)}
\end{array}\right]\left[\begin{array}{c}
X_{1} \\
X_{2} \\
\vdots \\
X_{3 n}
\end{array}\right] \equiv \widetilde{H} \mathbf{X}=0,
$$

where $\widetilde{H}$ is a $3 n \times 3 n$ matrix and its components are

$$
\begin{aligned}
& H_{1, \mathbf{G}}^{(l)}=\left[\begin{array}{c}
\left(C_{\mathbf{G}-\mathbf{G}^{\prime}}^{35}+C_{\mathbf{G}-\mathbf{G}^{\prime}}^{45}+C_{\mathbf{G}-\mathbf{G}^{\prime}}^{55}\right)\left(k_{1}+G_{1}^{\prime}\right) \boldsymbol{\varepsilon}_{\mathbf{G}^{\prime}}^{3(l)}+ \\
\left(C_{\mathbf{G}-\mathbf{G}^{\prime}}^{35}+C_{\mathbf{G}-\mathbf{G}^{\prime}}^{45}+C_{\mathbf{G}-\mathbf{G}^{\prime}}^{55}\right)\left(k_{z}^{(l)}\right) \boldsymbol{\varepsilon}_{\mathbf{G}^{\prime}}^{1(l)}
\end{array}\right], \\
& H_{2, \mathbf{G}}^{(l)}=\left[\begin{array}{c}
\left(C_{\mathbf{G}-\mathbf{G}^{\prime}}^{34}+C_{\mathbf{G}-\mathbf{G}^{\prime}}^{44}+C_{\mathbf{G}-\mathbf{G}^{\prime}}^{54}\right)\left(k_{2}+G_{2}^{\prime}\right) \boldsymbol{\varepsilon}_{\mathbf{G}^{\prime}}^{3(l)}+ \\
\left(C_{\mathbf{G}-\mathbf{G}^{\prime}}^{34}+C_{\mathbf{G}-\mathbf{G}^{\prime}}^{44}+C_{\mathbf{G}-\mathbf{G}^{\prime}}^{54}\right)\left(k_{z}^{(l)}\right) \boldsymbol{\varepsilon}_{\mathbf{G}^{\prime}}^{(l)}
\end{array}\right],
\end{aligned}
$$

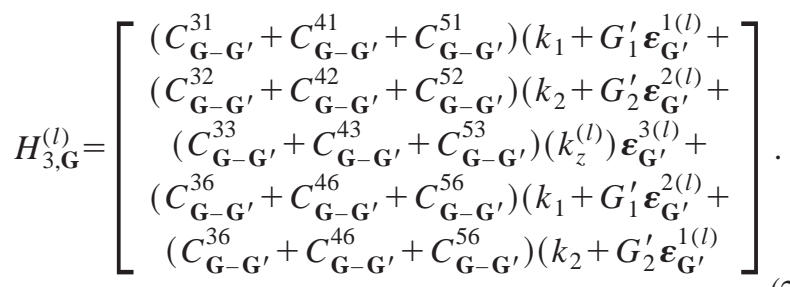

For the existence of a nontrivial solution of $X_{l}$, the following condition must be satisfied, i.e.,

$$
\operatorname{det}(\widetilde{H})=0 .
$$

Equation (23) is the dispersion relation for surface waves propagating in two-dimensional phononic crystals with both the filling material and the background material belong to the triclinic system. The relative magnitude of the eigenvectors $X_{l}$ can be obtained by substituting $k_{z}$ and $\omega$, which satisfy Eq. (23), into Eq. (19).

\section{NUMERICAL EXAMPLES}

The Fourier coefficients, $\rho_{\mathbf{G}}$ and $C_{\mathbf{G}}^{i j m n}$, in Eqs. (4) and (5), can be expressed as

$$
\alpha_{\mathbf{G}}=\left\{\begin{array}{cc}
\alpha_{A} f+\alpha_{B}(1-f) & \text { for } \mathbf{G}=0, \\
\left(\alpha_{A}-\alpha_{B}\right) F_{\mathbf{G}} & \text { for } \mathbf{G} \neq 0,
\end{array}\right.
$$

where $\alpha=\left(\rho, C^{i j m n}\right), f$ is the filling fraction that defines the cross-sectional area of a cylinder relative to a unit-cell area, and $F_{\mathbf{G}}$ is called the structure function defined as

$$
F_{\mathbf{G}}=A_{c}^{-1} \int_{A_{c}} d^{2} x e^{-i \mathbf{G} \cdot \mathbf{x}} .
$$

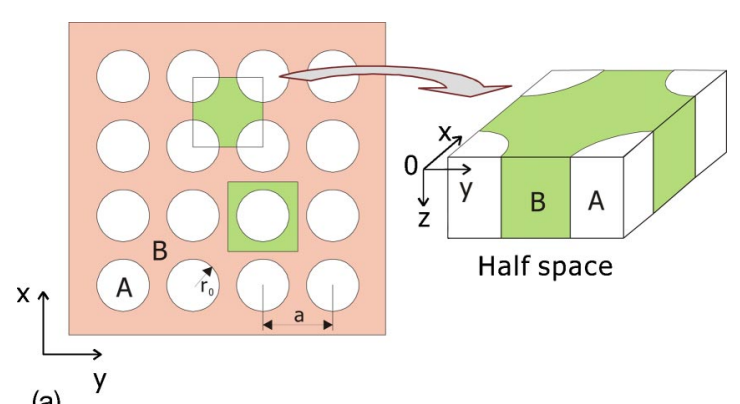

(a)

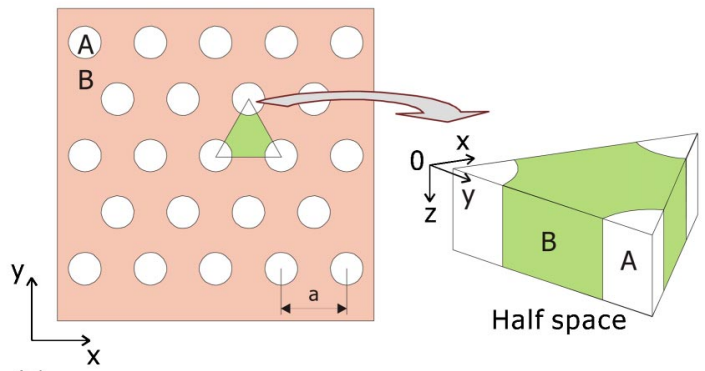

(b)

FIG. 1. Phononic structures with the square lattice (a) and the hexagonal lattice (b). 


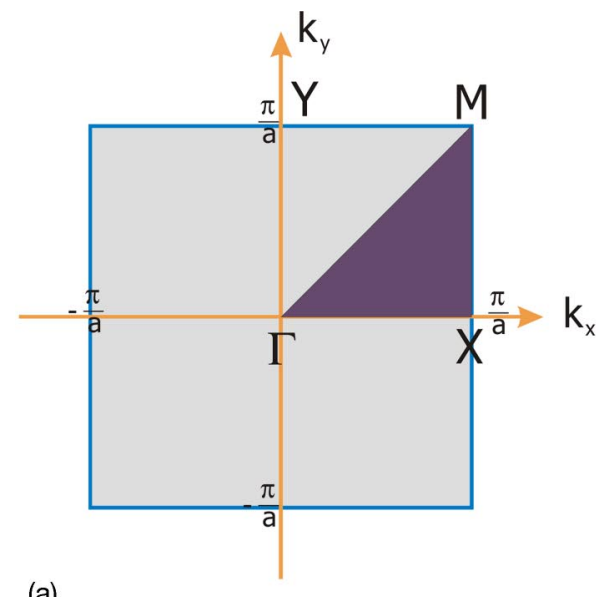

(a)

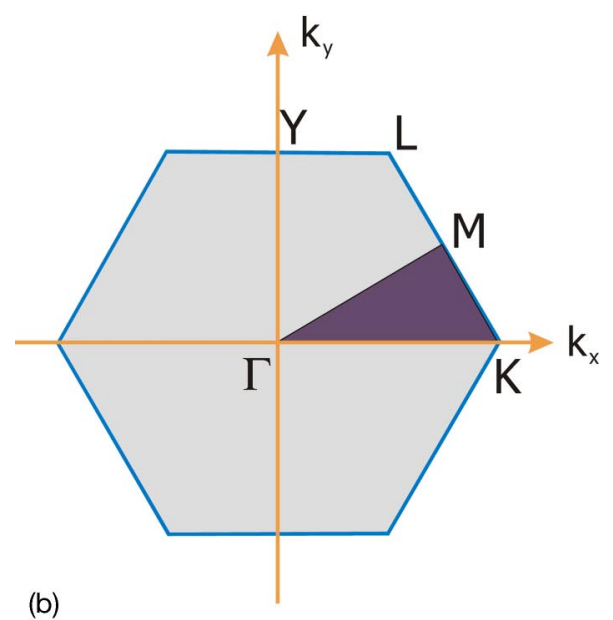

FIG. 2. Brillouin zone of the square lattice (a) and the hexagonal lattice (b).

In the above equation, $A_{c}$ is the cross section area of the filling structure. The structure function is then reduced to

$$
F_{\mathbf{G}}=\frac{2 f J_{1}\left(G r_{0}\right)}{G r_{0}}
$$

with $J_{1}(x)$ a first order Bessel function.

In this paper, phononic structures with square lattice and hexagonal lattice are considered. These lattices consist of circular cylinders $(A)$ embedded in a background material (B) forming two-dimensional lattices with lattice spacing $a$ as shown in Fig. 1(a) (square lattice) and Fig. 1(b) (hexagonal lattice). Figures 2(a) and 2(b) are the Brillouin regions of the square lattice and the hexagonal lattice, respectively. In
TABLE I. The elastic properties of the materials utilized in the examples (I).

\begin{tabular}{cccccccc}
\hline \hline & & \multicolumn{6}{c}{ Elastic constants $\left(\times 10^{10} \mathrm{~N} / \mathrm{m}^{2}\right)$} \\
\cline { 5 - 8 } Material & Symmetry & $\begin{array}{c}\text { Density } \\
\left(\mathrm{kg} / \mathrm{m}^{3}\right)\end{array}$ & $C_{11}$ & $C_{12}$ & $C_{13}$ & $C_{33}$ & $C_{44}$ \\
\hline $\mathrm{Ni}$ & Isotropic & 8905 & 32.4 & 16.4 & & 8 \\
$\mathrm{Al}$ & Isotropic & 2695 & 11.1 & 6.1 & & 2.5 \\
$\mathrm{AlAs}$ & Cubic & 3760 & 12.02 & 5.70 & & 5.89 \\
$\mathrm{GaAs}$ & Cubic & 5360 & 11.88 & 5.38 & & 5.94 \\
$\mathrm{ZnO}$ & Hexagonal & 5680 & 20.97 & 12.11 & 10.51 & 21.09 & 4.247 \\
\hline \hline
\end{tabular}

the square lattice, the reciprocal lattice vector is $\mathbf{G}$ $=\left(2 \pi N_{1} / a, 2 \pi N_{2} / a\right)$, where $N_{1}, N_{2}=0, \pm 1, \pm 2, \ldots$ and the filling fraction is $f=\left(\pi r_{0}^{2}\right) / a^{2}$. The irreducible part of the Brillouin zone of a square lattice is shown in Fig. 2(a), which is a triangle with vertices $\Gamma, X, M$. The reciprocal lattice vector of a hexagonal lattice is $\mathbf{G}=\left(2 \pi N_{1} / a, 2 \pi\left(2 N_{2}\right.\right.$ $\left.-N_{1}\right) / \sqrt{3} a$ ), where $N_{1}, N_{2}=0, \pm 1, \pm 2, \ldots$ and the filling fraction is $f=\left(2 \pi r_{0}^{2}\right) / \sqrt{3} a^{2}$. The irreducible part of the Brillouin zone of a hexagonal lattice is shown in Fig. 2(b), which is a triangle with vertices $\Gamma, K, M$. The elastic properties of the materials utilized in the following examples are adopted from Ref. 17 and listed in Table I and Table II.

\section{A. Isotropic materials: $\mathbf{A l} / \mathrm{Ni}$ square lattice}

Consider a phononic structure consisting of aluminum (Al) circular cylinders embedded in a background material of $\mathrm{Ni}$ forming a two-dimensional square lattice with lattice spacing $a$. Figure 3 shows the dispersion relations along the boundaries of the irreducible part of the Brillouin zone with filling ratio $f=0.6$. The vertical axis is the normalized frequency $\omega^{*}=\omega a / C_{t}$ and the horizontal axis is the reduced wave number $k^{*}=k a / \pi$. As the elastic waves propagate along the $x$ axis, the nonvanishing displacement of the shear horizontal mode, shear vertical mode and longitudinal mode are $u_{y}, u_{z}$, and $u_{x}$ respectively. For the sequence modes appear, we denominate the same type mode as the fundamental, the first and the second modes et al. For example, in Fig. 3 , the thin solid lines represent the $\mathrm{SV}$ bulk acoustic modes (the fundamental mode is $\mathrm{SV}_{0}$ and the first mode is $\mathrm{SV}_{1}$ ), and the square symbols are those for the longitudinal acoustic mode $(\mathrm{L})$. The thin dashed line represents the fundamental shear horizontal mode $\mathrm{SH}_{0}$, while the lines with "+" symbols represent the first shear horizontal mode $\mathrm{SH}_{1}$. In Fig. 3, for bulk mode propagation along the $x$ direction $(\Gamma-X)$, there are clear band gaps exist for the $\mathrm{SV}\left(\omega^{*}\right.$

TABLE II. The elastic properties of the materials utilized in the examples (II).

\begin{tabular}{lccccccc}
\hline \hline & & & \multicolumn{5}{c}{ Elastic constants $\left(\times 10^{10} \mathrm{~N} / \mathrm{m}^{2}\right)$} \\
\cline { 3 - 7 } Material & Symmetry & $\begin{array}{c}\text { Density } \\
\left(\mathrm{kg} / \mathrm{m}^{3}\right)\end{array}$ & $C_{11}$ & $C_{12}$ & $C_{13}$ & $C_{23}$ & $C_{22}$ \\
\hline \multirow{2}{*}{$\mathrm{Ba}_{2} \mathrm{NaNb}_{5} \mathrm{O}_{15}$} & \multirow{2}{*}{ Orthorhombic } & \multirow{2}{*}{5300} & 23.9 & 10.4 & 5.0 & 5.2 & 24.4 \\
& & & $C_{33}$ & $C_{44}$ & $C_{55}$ & $C_{66}$ & \\
& & 13.5 & 6.5 & 6.6 & 7.6 \\
\hline \hline
\end{tabular}




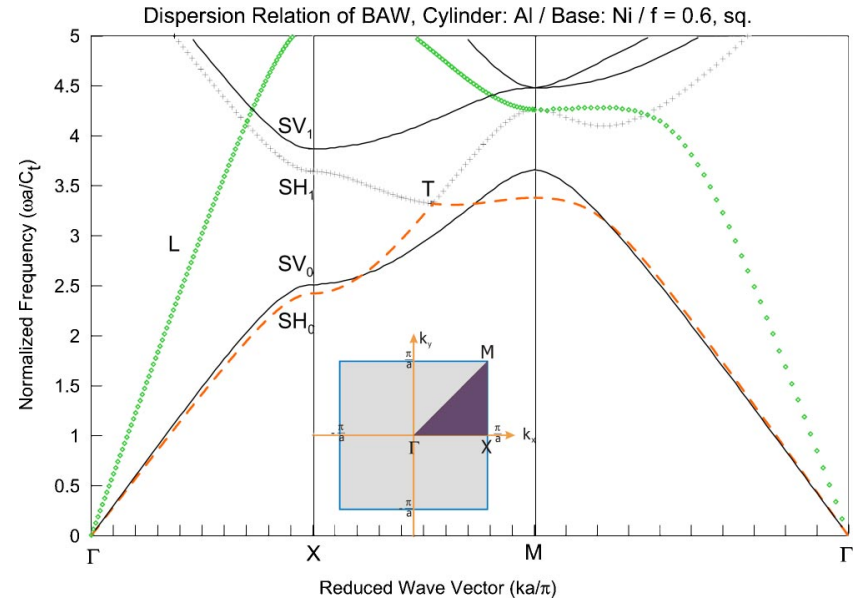

FIG. 3. Dispersion relations of all bulk modes ( $\mathrm{Al} / \mathrm{Ni}, f=0.6$, sq.).

$=1.36)$ and $\mathrm{SH}\left(\omega^{*}=1.22\right)$ bulk modes and the phase velocities of the SV mode (thin solid line) are larger than those of the SH mode. The boundary of the Brillouin zone $X-M$ of Fig. 3 represents the dispersion of the bulk waves with propagating direction varied $0^{\circ}-45^{\circ}$ counterclockwise away from $x$ direction. At a first glance, it seems that the dispersion curves of the fundamental and the first shear horizontal modes, $\mathrm{SH}_{0}$ and $\mathrm{SH}_{1}$, make a cross at point $T$. However, detail calculation around the point $T$ (Fig. 4) shows that the fundamental and the first shear horizontal modes do not cross over, instead, these two modes bend away from each other. The corresponding wave propagating direction of point $T$ is about $28.44^{\circ}$.

To understand further the peculiar behavior of the shear horizontal modes at point $T$, we calculated the displacement fields of the $\mathrm{SH}_{0}$ and $\mathrm{SH}_{1}$ modes along all the boundaries of the Brillouin zone as those shown in Figs. 5 and 6 . In the $\Gamma-X$ section, the nonvanishing displacement of the shear horizontal mode is $u_{y}$ as expected and it vanishes at the band gap point $X$. As the propagating direction move away from the $X$ point, one finds that $u_{y}$ remains very small; however, $u_{x}$ increases gradually until the point $T$. At this particular propagating direction, $u_{x}$ suddenly jumps to a very small

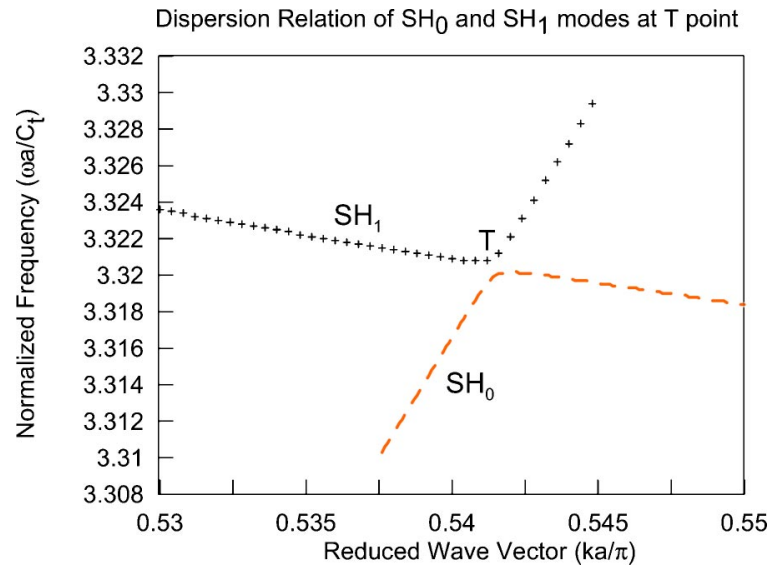

FIG. 4. Detail calculation around the point $T$.

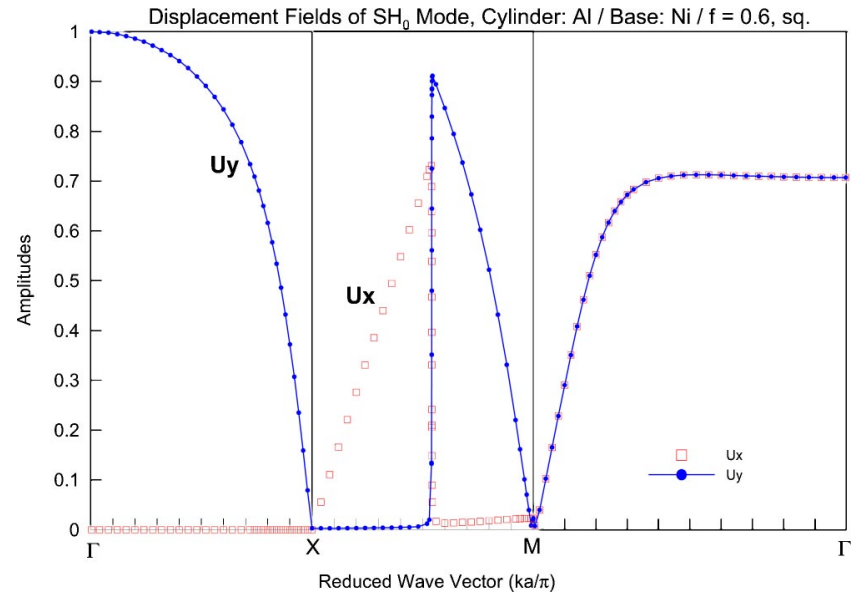

FIG. 5. Displacement fields of the $\mathrm{SH}_{0}$ modes along all the boundaries of the Brillouin zone ( $\mathrm{Al} / \mathrm{Ni}, f=0.6$, sq.).

value, instead, $u_{y}$ jumps from a small value to a finite value and then decays to zero at the band gap point $M$. Figure 6 shows the similar calculated result of the displacement field of the $\mathrm{SH}_{1}$ mode. In contrast to the $\mathrm{SH}_{0}$, at point $X, u_{y}$ decreases from a finite value gradually until the point $T$, then a sudden jump to a very small value. At point $T, u_{x}$ jump up suddenly from a very small value to a finite value. With Figs. 5 and 6 , we find clearly the mode interchange appears at the sharp bend of the dispersion curve (point $T$ in Fig. 4). It is worth noting that in both of the $\mathrm{SH}_{0}$ and $\mathrm{SH}_{1}$ modes (Figs. 5 and 6), the magnitudes of the displacements $u_{x}$ and $u_{y}$ on the $\Gamma-M$ section are equal due to the symmetry of the lattice arrangements.

Figure 7 shows the dispersion relation of the surface wave modes in the phononic structure with $\mathrm{Al} / \mathrm{Ni}$ square lattice. The solid circles represent the dispersion relations of the surface wave modes (SAW) and the open circles are those for the pseudosurface wave (PSAW) modes. For later convenience in discussing the interaction of the surface wave and bulk wave, the dispersion of the bulk modes are also shown in the figure. Result showed that as the normalized frequency of the surface wave mode lies between the $\mathrm{SH}_{0}$ and $\mathrm{SH}_{1}$

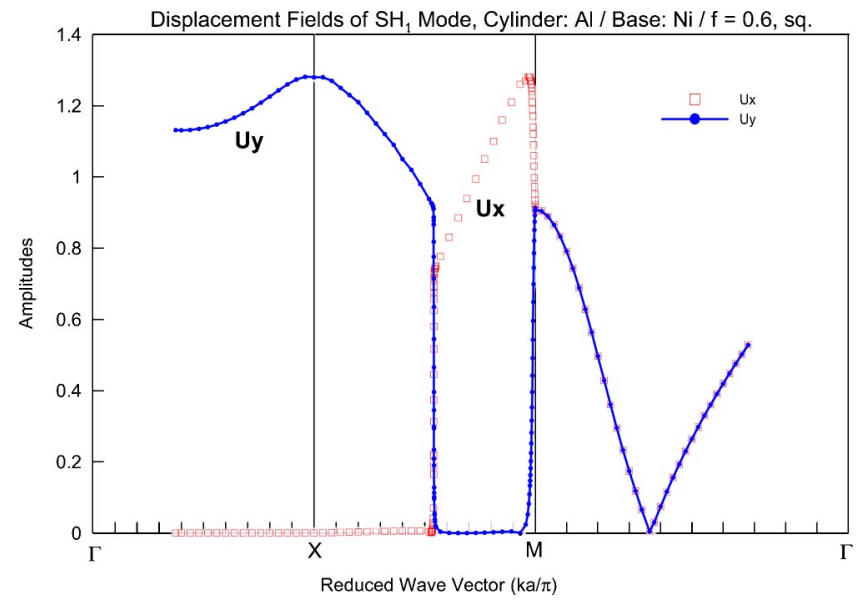

FIG. 6. Displacement fields of the $\mathrm{SH}_{1}$ modes along all the boundaries of the Brillouin zone ( $\mathrm{Al} / \mathrm{Ni}, f=0.6$, sq.). 


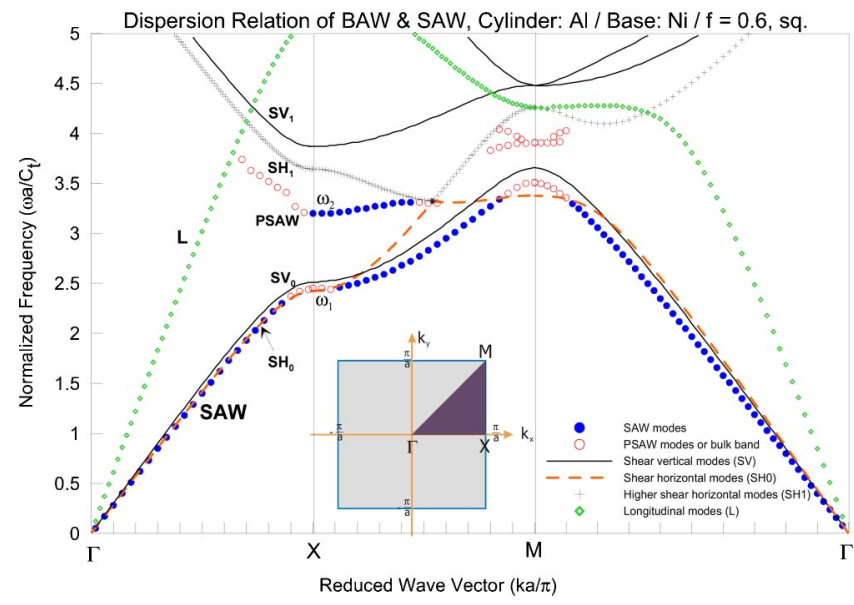

FIG. 7. Dispersion relations of BAW and SAW modes (Al/Ni, $f=0.6$, sq.).

modes, the surface wave degenerates into the pseudosurface wave mode. Unlike the normal surface wave mode, the displacement of the pseudosurface wave mode does not decay to zero at large depth. In the present case, one finds that at point $X$, instead of the SAW-SAW band gap, there only exists SAW-PSAW band gap. It is worth noting that in Fig. 7, from $X$ to $M$, the higher SAW mode ceased approximately at the $T$ point where the sharp bend of the bulk SH mode occurs.

To test the convergence of the plane wave expansion method, we chose the fundamental shear vertical mode $\mathrm{SV}_{0}$ as an example and calculated the dispersion curves with different number of the reciprocal lattice vectors. The testing results showed that as the reciprocal lattice vector number increases to a specific value, the normalized frequency of the $\mathrm{SV}_{0}$ mode almost converges to a constant value. For example, the difference between the results using 49 RLV and those using $169 \mathrm{RLV}$ is about $0.5 \%$. The convergence test for the SAW mode also showed a similar trend as that of the bulk acoustic wave. For computing time consideration, we used $49 \mathrm{RLV}$ in all the calculations conducted in this paper.

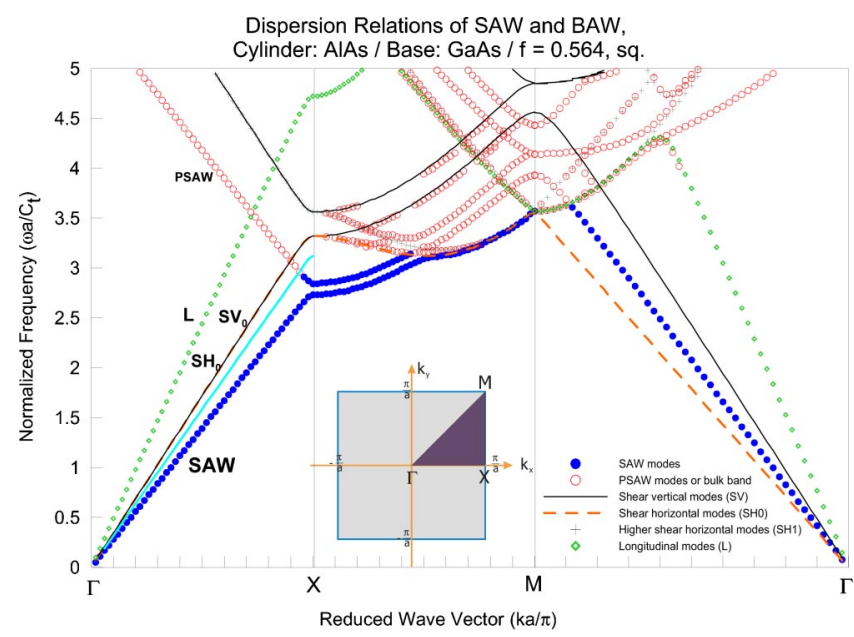

FIG. 8. Dispersion relations of BAW and SAW modes (AlAs/ GaAs, $f=0.564$, sq.).

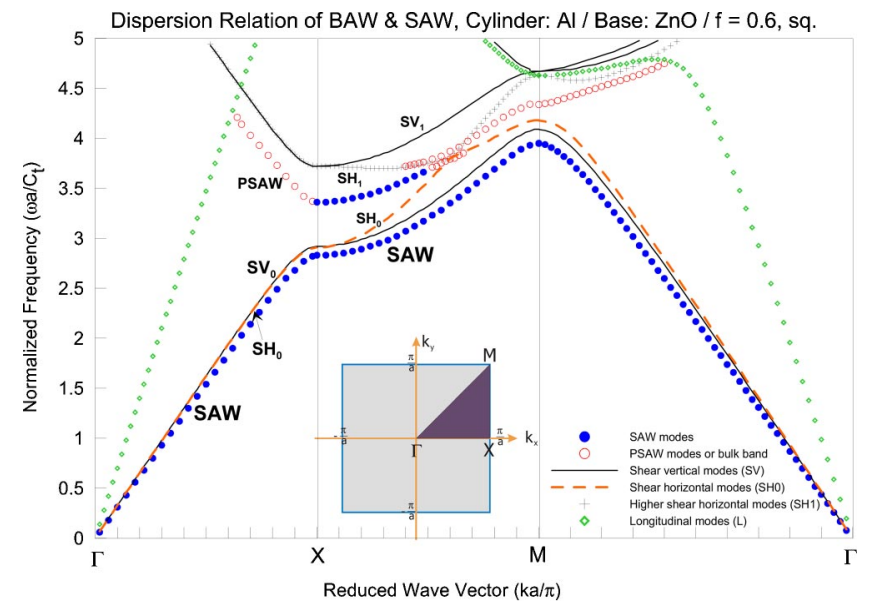

FIG. 9. Dispersion relations of BAW and SAW modes $(\mathrm{Al} / \mathrm{ZnO}$, $f=0.6$, sq.).

\section{B. Cubic materials: AlAs/GaAs square lattice}

In Ref. 7, the dispersion curves of the surface waves propagate in a two-dimensional square lattice consisting of circular cylinders of AlAs embedded in a background material of GaAs with filling fraction $f=0.564$ have been reported. Shown in Fig. 8 is dispersion curves reproduced from this study, one finds that the result is exactly the same as that in Ref. 7. Details of the discussions of bulk and surface wave dispersions in this phononic structure can be found therein.

\section{Hexagonal materials:}

$\mathrm{Al} / \mathrm{ZnO}$ square lattice and hexagonal lattice

In this subsection, we consider a phononic structure consisting of circular cylinders of $\mathrm{Al}$ embedded in a background material of $\mathrm{ZnO}$ forming a two-dimensional square lattice. The material of the filling cylinders is isotropic aluminum and the base material $\mathrm{ZnO}$ is in hexagonal symmetry. In the following calculations, the $x-y$ plane is parallel to the (001) plane and the $x$ axis is parallel to the [100] direction of $\mathrm{ZnO}$ and the filling ratio is 0.6. Shown in Fig. 9 are the dispersion curves for both of the bulk modes and surface acoustic modes. Similar to the case of the isotropic material shown in Fig. 3, the thin solid lines represent the SV bulk acoustic modes $\left(\mathrm{SV}_{0}\right.$ and $\left.\mathrm{SV}_{1}\right)$, and the square symbols are those for the longitudinal acoustic mode $(L)$. The thin dashed line

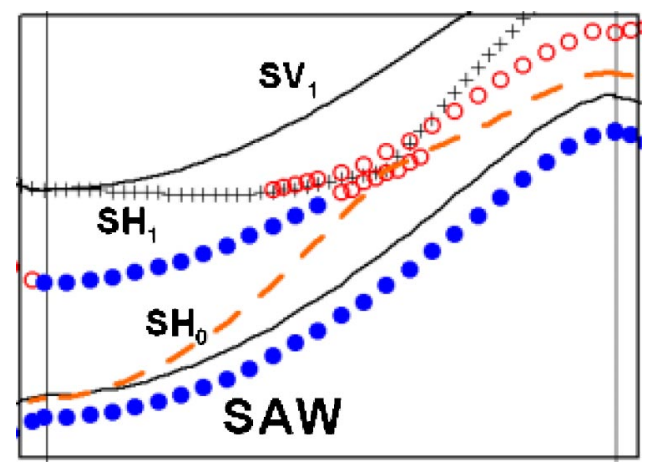

FIG. 10. An enlarged plot of Fig. 9 around the sharp bends. 
represents the fundamental shear horizontal mode $\mathrm{SH}_{0}$, while the lines with "+" symbols represent the first shear horizontal mode $\mathrm{SH}_{1}$. The dispersion of the bulk modes shows similar characteristics as that of the isotropic $\mathrm{Al} / \mathrm{Ni}$ case. Except that in the $\Gamma-X$ section, the higher and lower $\mathrm{SV}$ and SH modes are almost overlapped, and therefore the band gap widths of these two modes are similar at the Brillouin boundary point $X$. In the $X-M$ section, the dispersion curves of the $\mathrm{SH}_{0}$ and $\mathrm{SH}_{1}$ modes still approaching to each other, however, the bends at the closest point are of not so sharp as those in the $\mathrm{Al} / \mathrm{Ni}$ case.

In Fig. 9, unlike the $\mathrm{Al} / \mathrm{Ni}$ phononic square lattice shown in Fig. 8, we found that the lower SAW mode exists through all the boundaries of the Brillouin zone. No PSAW exists in this branch for in this case, all the SAW velocities are smaller than the bulk shear and longitudinal wave velocities. We note that in the $\Gamma-X$ section, the higher SAW mode becomes PSAW, while in the $X-M$ section, it preserves the SAW characteristics and extends from $X$ to approximately the sharp bend position, and then, degenerates into the PSAW for a small section as shown in the figure. On the other hand, the higher surface acoustic wave extends from the $M$ point belongs to the PSAW as shown in Fig. 9. It ends at approximately the sharp bends of the $\mathrm{SH}_{1}$ mode. An enlarged plot of Fig. 9 around the sharp bends is shown in Fig. 10 . One finds clearly that the PSAW mode extends from the $X$ point is tangentially merged into the $\mathrm{SH}_{0}$ mode and ceased at the intersection point. On the other hand, the PSAW mode extends from the $M$ point is tangentially merged into the $\mathrm{SH}_{1}$ mode and ceased at the intersection point.

Shown in Fig. 11 are the dispersion curves of the bulk modes and surface acoustic modes of the $\mathrm{Al} / \mathrm{ZnO}$ phononic structure with hexagonal lattice. For the shear horizontal modes $\mathrm{SH}_{0}$ (thin dashed line) and $\mathrm{SH}_{1}$ (+ symbols), unlike that of the square lattice, we find no sharp bend occurs in the $K-M$ section. Therefore, there is no $\mathrm{SH}$ mode interchange exists in this phononic structure with hexagonal lattice. From Fig. 11, we found that at $K$ point, there is no surface wave band gap existed. However, along the $\Gamma-M$ boundary, a SAW-PSAW band gap exists at the boundary point $M$.

\section{Orthorhombic materials: $\mathrm{Al} / \mathrm{Ba}_{2} \mathrm{NaNb}_{5} \mathrm{O}_{15}$ square lattice and hexagonal lattice}

In this subsection, we consider a phononic structure consisting of circular cylinders of $\mathrm{Al}$ embedded in a background material of barium sodium niobate $\left(\mathrm{Ba}_{2} \mathrm{NaNb}_{5} \mathrm{O}_{15}\right)$ forming a two-dimensional square lattice. The material of the filling cylinders is isotropic aluminum and the base material is barium sodium niobate with orthorhombic symmetry. In the following calculations, the $x-y$ plane is parallel to the (001) plane and the $x$ axis is parallel to the [100] direction of barium sodium niobate. The filling fraction is $f=0.6$.

Shown in Fig. 12 are the dispersion curves for both of the bulk modes and surface acoustic modes. The surface wave dispersion curves along $\Gamma-X$ and $\Gamma-M$ directions lie below the dispersion curves of transverse waves and degenerate to PSAW along the boundaries of $X-M$ and $\Gamma-M$. At one of the vertex of the Brillouin region, $M$, there is a clear frequency

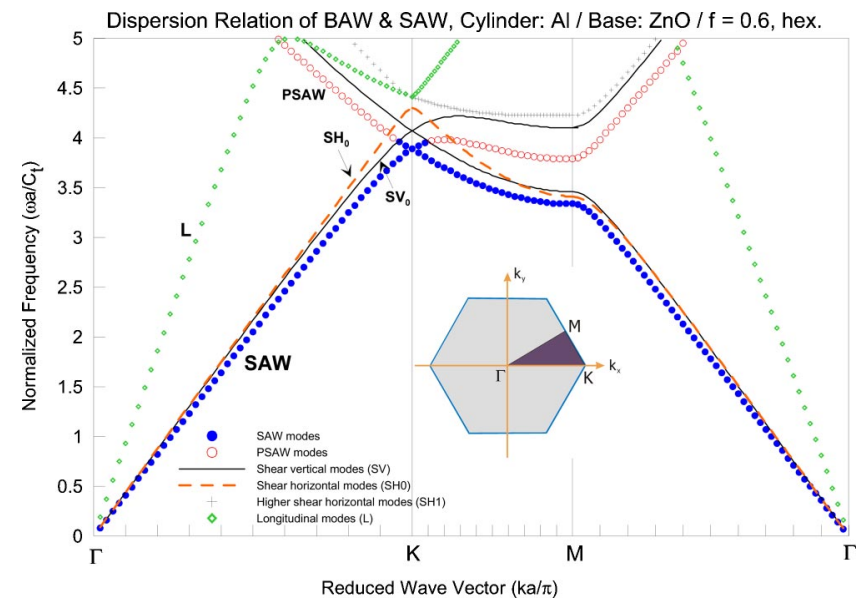

FIG. 11. Dispersion relations of BAW and SAW modes (Al/ $\mathrm{ZnO}, f=0.6$, hex.).

band gap with nondimensional width of 0.26 between PSAW and PSAW. At the vertex $X$, we found a frequency band gap between SAW and SAW with nondimensional width of 0.52 . Similar to the isotropic $\mathrm{Al} / \mathrm{Ni}$ square lattice, the surface wave and higher SAW mode continues to exist along $X-M$ boundary up to about $39.35^{\circ}$ and $24.70^{\circ}$ rotated from $X$ point in Fig. 12.

For some materials with symmetry higher (and equal to) than hexagonal symmetry, the $x-y$ plane is isotropic for all the propagation modes. The irreducible part of the Brillouin zone of a square lattice is an isosceles right-angled triangle showed in Fig. 2(a). But the material with orthorhombic symmetry is anisotropic in the $x-y$ plane; the dispersion relations of propagation along $x$ axis and $y$ axis are different. The irreducible part of the Brillouin zone of a square lattice is extended to a square with vertices $\Gamma, X, M, Y$ shown in Fig. 2(a). However, due to the small difference between $C_{11}$ and $C_{22}$ and between $C_{13}$ and $C_{23}$, we found that the differences of dispersion relations along $\Gamma-X$ and $\Gamma-Y$ are very small in this case, therefore, only $\Gamma, X, M$ are considered in Fig. 12.

To further investigate the anisotropic effect on the dispersion of a phononic structure, we considered the $Y$-cut barium

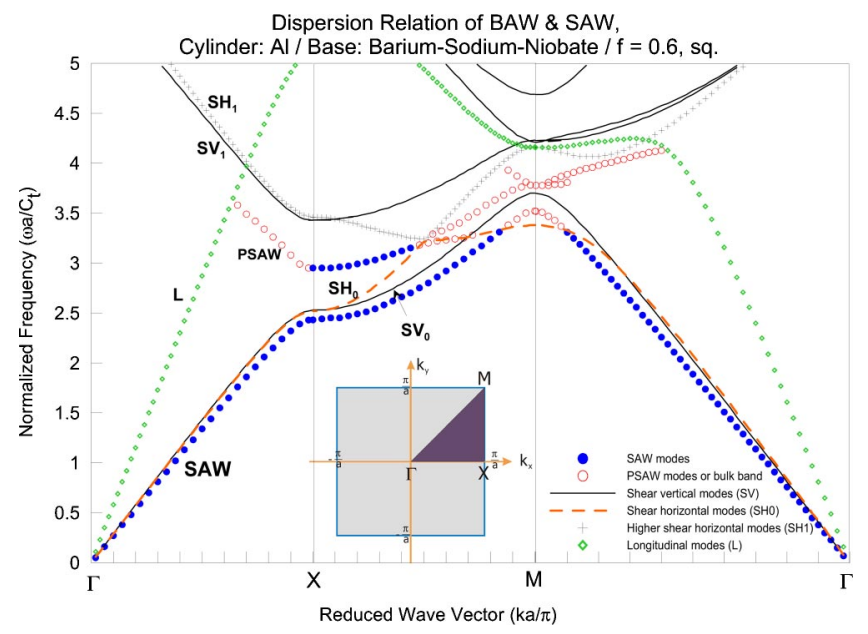

FIG. 12. Dispersion relations of BAW and SAW modes (Al/ barium sodium niobate, $f=0.6$, sq.). 


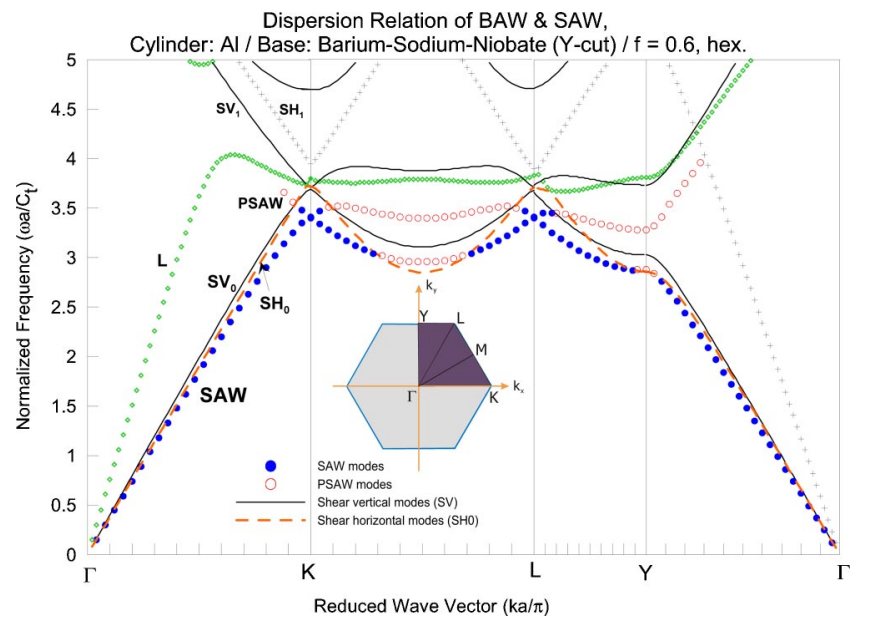

FIG. 13. Dispersion relations of BAW and SAW modes [A1/ barium sodium niobate (Y cut), $f=0.6$, hex.].

sodium niobate as the background material. Shown in Fig. 13 are the dispersion curves of the bulk modes and surface acoustic modes of the $\mathrm{Al} / \mathrm{barium}$ sodium niobate ( $Y$-cut) phononic structure with hexagonal lattice. According to the discussions in the above paragraph, the irreducible part of the Brillouin zone of a hexagonal lattice for anisotropic materials must be extended to the first quadrant in original hexagonal lattice with vertices $\Gamma, K, L, Y$ shown in the inset of Fig. 13.

For the longitudinal modes $L$ (square symbols), unlike that of the square lattice, we find that band gap occurs in the $\Gamma-K$ section (the width is 0.91 ). It is seen that the dispersion relations in $K-L$ section are almost symmetric respect to the center of the section ( $M$ point). It is worth noting that in the $L-Y$ section, sharp bends of the dispersion curves occur between $\mathrm{SH}_{1}$ and $L$ modes and between $L$ and $\mathrm{SH}_{0}$ modes. Therefore, there are mode interchanges ( $\mathrm{SH}$ and $L$ ) exist in this phononic structure with hexagonal lattice. In the $\Gamma-Y$ section, the waves propagation are along $y$ axis, the displacement fields of longitudinal and shear horizontal modes are $u_{y}$ and $u_{x}$, respectively.

From Fig. 13, we find that at $K$ point, there is no surface wave band gap existed. However, along the $\Gamma-Y$ boundary, a PSAW-PSAW band gap exists at the boundary point $Y$. The phenomenon shows the characteristics of an anisotropic material.

\section{CONCLUSION}

In this paper, we studied the phononic band gaps of surface waves in two-dimensional phononic structures consist of general anisotropic materials. The explicit formulations of the plane harmonic bulk wave and the surface wave dispersion relations in such a general phononic structure are derived based on the plane wave expansion method. Twodimensional phononic structures with either the square or the hexagonal lattice are considered in the numerical examples. Band gap characteristics of the phononic structures with different anisotropic background materials (isotropic, cubic, hexagonal and orthorhombic) are calculated and discussed. It is worth noting that some of the crossing over of the dispersion curves (apparently) is indeed sharp bends of the dispersion curves. Around this sharp bend area, the mode exchange suddenly. Results of this paper can serve as a basis for both numerical and experimental investigations of phononic crystal structures consist of general anisotropic materials.

\section{ACKNOWLEDGMENTS}

The authors thank the National Science Council of Taiwan (NSC91-2212-E-002-057), Academia Sinica of Taiwan and the NTU-ITRI center for financial support.

\section{APPENDIX}

The expressions of the 27 matrices in Eq. (7) are

$$
\begin{gathered}
M_{\mathbf{G}, \mathbf{G}^{\prime}}^{(1)}=\left[\begin{array}{c}
\omega^{2} \rho_{\mathbf{G}^{-}-\mathbf{G}^{\prime}} \\
-\left(\mathbf{G}_{1}+k_{1}\right)\left(\mathbf{G}_{1}^{\prime}+k_{1}\right) C_{\mathbf{G}-\mathbf{G}^{\prime}}^{11}-\left(\mathbf{G}_{1}+k_{1}\right)\left(\mathbf{G}_{2}^{\prime}+k_{2}\right) C_{\mathbf{G}-\mathbf{G}^{\prime}}^{16} \\
-\left(\mathbf{G}_{2}+k_{2}\right)\left(\mathbf{G}_{1}^{\prime}+k_{1}\right) C_{\mathbf{G}-\mathbf{G}^{\prime}}^{16}-\left(\mathbf{G}_{2}+k_{2}\right)\left(\mathbf{G}_{2}^{\prime}+k_{2}\right) C_{\mathbf{G}-\mathbf{G}^{\prime}}^{66}
\end{array}\right], \\
M_{\mathbf{G}, \mathbf{G}^{\prime}}^{(2)}=\left[\begin{array}{c}
\omega^{2} \rho_{\mathbf{G}^{\prime}-\mathbf{G}^{\prime}} \\
-\left(\mathbf{G}_{1}+k_{1}\right)\left(\mathbf{G}_{1}^{\prime}+k_{1}\right) C_{\mathbf{G}-\mathbf{G}^{\prime}}^{66}-\left(\mathbf{G}_{1}+k_{1}\right)\left(\mathbf{G}_{2}^{\prime}+k_{2}\right) C_{\mathbf{G}^{\prime}-\mathbf{G}^{\prime}}^{26} \\
-\left(\mathbf{G}_{2}+k_{2}\right)\left(\mathbf{G}_{1}^{\prime}+k_{1}\right) C_{\mathbf{G}-\mathbf{G}^{\prime}}^{26}-\left(\mathbf{G}_{2}+k_{2}\right)\left(\mathbf{G}_{2}^{\prime}+k_{2}\right) C_{\mathbf{G}^{2}-\mathbf{G}^{\prime}}^{22}
\end{array}\right], \\
M_{\mathbf{G}, \mathbf{G}^{\prime}}^{(3)}=\left[\begin{array}{c}
\omega^{2} \rho_{\mathbf{G}^{-}-\mathbf{G}^{\prime}} \\
-\left(\mathbf{G}_{1}+k_{1}\right)\left(\mathbf{G}_{1}^{\prime}+k_{1}\right) C_{\mathbf{G}-\mathbf{G}^{\prime}}^{55}-\left(\mathbf{G}_{1}+k_{1}\right)\left(\mathbf{G}_{2}^{\prime}+k_{2}\right) C_{\mathbf{G}-\mathbf{G}^{\prime}}^{45} \\
-\left(\mathbf{G}_{2}+k_{2}\right)\left(\mathbf{G}_{1}^{\prime}+k_{1}\right) C_{\mathbf{G}-\mathbf{G}^{\prime}}^{45}-\left(\mathbf{G}_{2}+k_{2}\right)\left(\mathbf{G}_{2}^{\prime}+k_{2}\right) C_{\mathbf{G}-\mathbf{G}^{\prime}}^{44}
\end{array}\right], \\
S_{\mathbf{G}, \mathbf{G}^{\prime}}^{(1)}=\left[\begin{array}{c}
-\left(\mathbf{G}_{1}+k_{1}\right) C_{\mathbf{G}-\mathbf{G}^{\prime}}^{15}-\left(\mathbf{G}_{2}+k_{2}\right) C_{\mathbf{G}-\mathbf{G}^{\prime}}^{56} \\
-\left(\mathbf{G}_{1}^{\prime}+k_{1}\right) C_{\mathbf{G}-\mathbf{G}^{\prime}}^{15}-\left(\mathbf{G}_{2}^{\prime}+k_{2}\right) C_{\mathbf{G}^{5}-\mathbf{G}^{\prime}}^{56}
\end{array}\right],
\end{gathered}
$$




$$
\begin{aligned}
& S_{\mathbf{G}, \mathbf{G}^{\prime}}^{(2)}=\left[\begin{array}{l}
-\left(\mathbf{G}_{1}+k_{1}\right) C_{\mathbf{G}-\mathbf{G}^{\prime}}^{46}-\left(\mathbf{G}_{2}+k_{2}\right) C_{\mathbf{G}-\mathbf{G}^{\prime}}^{24} \\
-\left(\mathbf{G}_{1}^{\prime}+k_{1}\right) C_{\mathbf{G}-\mathbf{G}^{\prime}}^{46}-\left(\mathbf{G}_{2}^{\prime}+k_{2}\right) C_{\mathbf{G}-\mathbf{G}^{\prime}}^{24}
\end{array}\right], \\
& S_{\mathbf{G}, \mathbf{G}^{\prime}}^{(3)}=\left[\begin{array}{l}
-\left(\mathbf{G}_{1}+k_{1}\right) C_{\mathbf{G}-\mathbf{G}^{\prime}}^{35}-\left(\mathbf{G}_{2}+k_{2}\right) C_{\mathbf{G}-\mathbf{G}^{\prime}}^{34} \\
-\left(\mathbf{G}_{1}^{\prime}+k_{1}\right) C_{\mathbf{G}-\mathbf{G}^{\prime}}^{35}-\left(\mathbf{G}_{2}^{\prime}+k_{2}\right) C_{\mathbf{G}-\mathbf{G}^{\prime}}^{34}
\end{array}\right], \\
& N_{\mathbf{G}, \mathbf{G}^{\prime}}^{(1)}=-C_{\mathbf{G}-\mathbf{G}^{\prime}}^{55}, \quad N_{\mathbf{G}, \mathbf{G}^{\prime}}^{(2)}=-C_{\mathbf{G}-\mathbf{G}^{\prime}}^{44}, \quad N_{\mathbf{G}, \mathbf{G}^{\prime}}^{(3)}=-C_{\mathbf{G}-\mathbf{G}^{\prime}}^{33}, \\
& L_{\mathbf{G}, \mathbf{G}^{\prime}}^{(1)}=\left[\begin{array}{l}
-\left(\mathbf{G}_{1}+k_{1}\right)\left(\mathbf{G}_{1}^{\prime}+k_{1}\right) C_{\mathbf{G}-\mathbf{G}^{\prime}}^{16}-\left(\mathbf{G}_{1}+k_{1}\right)\left(\mathbf{G}_{2}^{\prime}+k_{2}\right) C_{\mathbf{G}-\mathbf{G}^{\prime}}^{12} \\
-\left(\mathbf{G}_{2}+k_{2}\right)\left(\mathbf{G}_{1}^{\prime}+k_{1}\right) C_{\mathbf{G}-\mathbf{G}^{\prime}}^{66}-\left(\mathbf{G}_{2}+k_{2}\right)\left(\mathbf{G}_{2}^{\prime}+k_{2}\right) C_{\mathbf{G}^{2}-\mathbf{G}^{\prime}}^{\prime 2}
\end{array}\right], \\
& L_{\mathbf{G}, \mathbf{G}^{\prime}}^{(2)}=\left[\begin{array}{l}
-\left(\mathbf{G}_{1}+k_{1}\right)\left(\mathbf{G}_{1}^{\prime}+k_{1}\right) C_{\mathbf{G}-\mathbf{G}^{\prime}}^{16}-\left(\mathbf{G}_{1}+k_{1}\right)\left(\mathbf{G}_{2}^{\prime}+k_{2}\right) C_{\mathbf{G}-\mathbf{G}^{\prime}}^{66} \\
-\left(\mathbf{G}_{2}+k_{2}\right)\left(\mathbf{G}_{1}^{\prime}+k_{1}\right) C_{\mathbf{G}-\mathbf{G}^{\prime}}^{21}-\left(\mathbf{G}_{2}+k_{2}\right)\left(\mathbf{G}_{2}^{\prime}+k_{2}\right) C_{\mathbf{G}-\mathbf{G}^{\prime}}^{26}
\end{array}\right], \\
& O_{\mathbf{G}, \mathbf{G}^{\prime}}^{(1)}=\left[\begin{array}{l}
-\left(\mathbf{G}_{1}+k_{1}\right) C_{\mathbf{G}-\mathbf{G}^{\prime}}^{14}-\left(\mathbf{G}_{2}+k_{2}\right) C_{\mathbf{G}-\mathbf{G}^{\prime}}^{46} \\
-\left(\mathbf{G}_{1}^{\prime}+k_{1}\right) C_{\mathbf{G}-\mathbf{G}^{\prime}}^{56}-\left(\mathbf{G}_{2}^{\prime}+k_{2}\right) C_{\mathbf{G}-\mathbf{G}^{\prime}}^{25}
\end{array}\right], \\
& O_{\mathbf{G}, \mathbf{G}^{\prime}}^{(2)}=\left[\begin{array}{l}
-\left(\mathbf{G}_{1}+k_{1}\right) C_{\mathbf{G}-\mathbf{G}^{\prime}}^{56}-\left(\mathbf{G}_{2}+k_{2}\right) C_{\mathbf{G}-\mathbf{G}^{\prime}}^{25} \\
-\left(\mathbf{G}_{1}^{\prime}+k_{1}\right) C_{\mathbf{G}-\mathbf{G}^{\prime}}^{14}-\left(\mathbf{G}_{2}^{\prime}+k_{2}\right) C_{\mathbf{G}-\mathbf{G}^{\prime}}^{46}
\end{array}\right], \\
& T_{\mathbf{G}, \mathbf{G}^{\prime}}^{(1)}=-C_{\mathbf{G}-\mathbf{G}^{\prime}}^{45}, \quad T_{\mathbf{G}, \mathbf{G}^{\prime}}^{(2)}=-C_{\mathbf{G}-\mathbf{G}^{\prime}}^{45}, \\
& U_{\mathbf{G}, \mathbf{G}^{\prime}}^{(1)}=\left[\begin{array}{l}
-\left(\mathbf{G}_{1}+k_{1}\right)\left(\mathbf{G}_{1}^{\prime}+k_{1}\right) C_{\mathbf{G}-\mathbf{G}^{\prime}}^{15}-\left(\mathbf{G}_{1}+k_{1}\right)\left(\mathbf{G}_{2}^{\prime}+k_{2}\right) C_{\mathbf{G}-\mathbf{G}^{\prime}}^{14} \\
-\left(\mathbf{G}_{2}+k_{2}\right)\left(\mathbf{G}_{1}^{\prime}+k_{1}\right) C_{\mathbf{G}-\mathbf{G}^{\prime}}^{56}-\left(\mathbf{G}_{2}+k_{2}\right)\left(\mathbf{G}_{2}^{\prime}+k_{2}\right) C_{\mathbf{G}^{-}-\mathbf{G}^{\prime}}^{46}
\end{array}\right], \\
& U_{\mathbf{G}, \mathbf{G}^{\prime}}^{(2)}=\left[\begin{array}{l}
-\left(\mathbf{G}_{1}+k_{1}\right)\left(\mathbf{G}_{1}^{\prime}+k_{1}\right) C_{\mathbf{G}-\mathbf{G}^{\prime}}^{56}-\left(\mathbf{G}_{1}+k_{1}\right)\left(\mathbf{G}_{2}^{\prime}+k_{2}\right) C_{\mathbf{G}-\mathbf{G}^{\prime}}^{46} \\
-\left(\mathbf{G}_{2}+k_{2}\right)\left(\mathbf{G}_{1}^{\prime}+k_{1}\right) C_{\mathbf{G}-\mathbf{G}^{\prime}}^{25}-\left(\mathbf{G}_{2}+k_{2}\right)\left(\mathbf{G}_{2}^{\prime}+k_{2}\right) C_{\mathbf{G}^{2}-\mathbf{G}^{\prime}}^{\prime \prime}
\end{array}\right], \\
& K_{\mathbf{G}, \mathbf{G}^{\prime}}^{(1)}=\left[\begin{array}{l}
-\left(\mathbf{G}_{1}+k_{1}\right) C_{\mathbf{G}-\mathbf{G}^{\prime}}^{13}-\left(\mathbf{G}_{2}+k_{2}\right) C_{\mathbf{G}-\mathbf{G}^{\prime}}^{36} \\
-\left(\mathbf{G}_{1}^{\prime}+k_{1}\right) C_{\mathbf{G}-\mathbf{G}^{\prime}}^{55}-\left(\mathbf{G}_{2}^{\prime}+k_{2}\right) C_{\mathbf{G}-\mathbf{G}^{\prime}}^{45}
\end{array}\right], \\
& K_{\mathbf{G}, \mathbf{G}^{\prime}}^{(2)}=\left[\begin{array}{l}
-\left(\mathbf{G}_{1}+k_{1}\right) C_{\mathbf{G}-\mathbf{G}^{\prime}}^{36}-\left(\mathbf{G}_{2}+k_{2}\right) C_{\mathbf{G}-\mathbf{G}^{\prime}}^{23} \\
-\left(\mathbf{G}_{1}^{\prime}+k_{1}\right) C_{\mathbf{G}-\mathbf{G}^{\prime}}^{45}-\left(\mathbf{G}_{2}^{\prime}+k_{2}\right) C_{\mathbf{G}-\mathbf{G}^{\prime}}^{44}
\end{array}\right], \\
& V_{\mathbf{G}, \mathbf{G}^{\prime}}^{(1)}=-C_{\mathbf{G}-\mathbf{G}^{\prime}}^{35}, \quad V_{\mathbf{G}, \mathbf{G}^{\prime}}^{(2)}=-C_{\mathbf{G}-\mathbf{G}^{\prime}}^{34}, \\
& W_{\mathbf{G}, \mathbf{G}^{\prime}}^{(1)}=\left[\begin{array}{l}
-\left(\mathbf{G}_{1}+k_{1}\right)\left(\mathbf{G}_{1}^{\prime}+k_{1}\right) C_{\mathbf{G}-\mathbf{G}^{\prime}}^{15}-\left(\mathbf{G}_{1}+k_{1}\right)\left(\mathbf{G}_{2}^{\prime}+k_{2}\right) C_{\mathbf{G}-\mathbf{G}^{\prime}}^{56} \\
-\left(\mathbf{G}_{2}+k_{2}\right)\left(\mathbf{G}_{1}^{\prime}+k_{1}\right) C_{\mathbf{G}-\mathbf{G}^{\prime}}^{14}-\left(\mathbf{G}_{2}+k_{2}\right)\left(\mathbf{G}_{2}^{\prime}+k_{2}\right) C_{\mathbf{G}-\mathbf{G}^{\prime}}^{46}
\end{array}\right], \\
& W_{\mathbf{G}, \mathbf{G}^{\prime}}^{(2)}=\left[\begin{array}{l}
-\left(\mathbf{G}_{1}+k_{1}\right)\left(\mathbf{G}_{1}^{\prime}+k_{1}\right) C_{\mathbf{G}-\mathbf{G}^{\prime}}^{56}-\left(\mathbf{G}_{1}+k_{1}\right)\left(\mathbf{G}_{2}^{\prime}+k_{2}\right) C_{\mathbf{G}-\mathbf{G}^{\prime}}^{25} \\
-\left(\mathbf{G}_{2}+k_{2}\right)\left(\mathbf{G}_{1}^{\prime}+k_{1}\right) C_{\mathbf{G}-\mathbf{G}^{\prime}}^{46}-\left(\mathbf{G}_{2}+k_{2}\right)\left(\mathbf{G}_{2}^{\prime}+k_{2}\right) C_{\mathbf{G}^{2}-\mathbf{G}^{\prime}}^{24}
\end{array}\right], \\
& J_{\mathbf{G}, \mathbf{G}^{\prime}}^{(1)}=\left[\begin{array}{l}
-\left(\mathbf{G}_{1}+k_{1}\right) C_{\mathbf{G}-\mathbf{G}^{\prime}}^{55}-\left(\mathbf{G}_{2}+k_{2}\right) C_{\mathbf{G}-\mathbf{G}^{\prime}}^{45} \\
-\left(\mathbf{G}_{1}^{\prime}+k_{1}\right) C_{\mathbf{G}-\mathbf{G}^{\prime}}^{13}-\left(\mathbf{G}_{2}^{\prime}+k_{2}\right) C_{\mathbf{G}-\mathbf{G}^{\prime}}^{36}
\end{array}\right], \\
& J_{\mathbf{G}, \mathbf{G}^{\prime}}^{(1)}=\left[\begin{array}{l}
-\left(\mathbf{G}_{1}+k_{1}\right) C_{\mathbf{G}-\mathbf{G}^{\prime}}^{45}-\left(\mathbf{G}_{2}+k_{2}\right) C_{\mathbf{G}-\mathbf{G}^{\prime}}^{44} \\
-\left(\mathbf{G}_{1}^{\prime}+k_{1}\right) C_{\mathbf{G}-\mathbf{G}^{\prime}}^{36}-\left(\mathbf{G}_{2}^{\prime}+k_{2}\right) C_{\mathbf{G}-\mathbf{G}^{\prime}}^{23}
\end{array}\right], \\
& X_{\mathbf{G}, \mathbf{G}^{\prime}}^{(1)}=-C_{\mathbf{G}-\mathbf{G}^{\prime}}^{35}, \quad X_{\mathbf{G}, \mathbf{G}^{\prime}}^{(2)}=-C_{\mathbf{G}-\mathbf{G}^{\prime}}^{34} .
\end{aligned}
$$

In the above equations, Voigt's notation has been used to rewrite $C_{\mathbf{G}}^{i j m n}$ as $C_{\mathbf{G}}^{I J}$. 
*Corresponding author. Email address: wutt@ndt.iam.ntu.edu.tw

${ }^{1}$ C. M. Soukoulis, Photonic Band Gaps and Localizations (Plenum, New York, 1993).

${ }^{2}$ J. D. Joannopoulos, R. D. Meade, and J. N. Winn, Photonic Crystals (Princeton University Press, Princeton, NJ, 1995).

${ }^{3}$ T. Aono and S. Tamura, Phys. Rev. B 58, 4838 (1998).

${ }^{4}$ R. E. Vines, J. P. Wolfe, and A. G. Every, Phys. Rev. B 60, 11871 (1999).

${ }^{5}$ R. E. Vines and J. P. Wolfe, Physica B 263-264, 567 (1999).

${ }^{6}$ A. G. Every, R. E. Vines, and J. P. Wolfe, Phys. Rev. B 60, 11755 (1999).

${ }^{7}$ Y. Tanaka and S. Tamura, Phys. Rev. B 58, 7958 (1998).

${ }^{8}$ Y. Tanaka and S. Tamura, Phys. Rev. B 60, 13294 (1999).

${ }^{9}$ M. S. Kushwaha, P. Halevi, L. Dobrzynski, and B. DjafariRouhani, Phys. Rev. Lett. 71, 2022 (1993).
${ }^{10}$ M. S. Kushwaha, P. Halevi, G. Martinez, L. Dobrzynski, and B. Djafari-Rouhani, Phys. Rev. B 49, 2313 (1994).

${ }^{11}$ M. Kafesaki and E. N. Economou, Phys. Rev. B 60, 11993 (1999).

${ }^{12}$ I. E. Psarobas and N. Stefanou, Phys. Rev. B 62, 278 (2000).

${ }^{13}$ Zhengyou Liu, C. T. Chan, and Ping Sheng, Phys. Rev. B 62, 2446 (2000).

${ }^{14}$ Jun Mei, Zhengyou Liu, Jing Shi, and Decheng Tian, Phys. Rev. B 67, 245107 (2003).

${ }^{15}$ D. Garica-Pablos, M. Sigalas, F. R. Montero de Espinosa, M. Kafesaki, and N. Garcia, Phys. Rev. Lett. 84, 4349 (2000).

${ }^{16}$ J. H. Wilkinson, The Algebraic Eigenvalue Problem (Clarendon, Oxford, 1965).

${ }^{17}$ B. A. Auld, Acoustic Fields and Waves in Solids, 2nd ed. (Kreiger, Malabar, FL, 1990), Vol. I. 